\title{
TRATAMENTO ENDOSCÓPICO DAS VARIZES ESOFÁGICAS UTILIZANDO ALÇAS PRÉ-ATADAS CONFECCIONADAS COM FIO DE POLIAMIDA
}

\author{
Francisco Susumu Corrêa KOYAMA, Kiyoshi HASHIBA, \\ Sansom Henrique BROMBERG e Carlos Alberto CAPPELANES
}

RESUMO - Racional - A ligadura com bandas elásticas tem se tornado o tratamento de escolha das varizes esofágicas, devido aos bons resultados e as menores taxas de complicações quando comparada à esclerose. Seu mecanismo de ação baseia-se na compressão mecânica do cordão varicoso com interrupção do fluxo sangüíneo e posterior trombose. Tendo como base este mecanismo de ação, desenvolveu-se um método alternativo no qual o cordão varicoso é ligado com uma alça confeccionada com fio de poliamida. Objetivo - Avaliar a viabilidade do tratamento das varizes esofágicas por método de ligaduras com alças pré-atadas confeccionadas com fio de poliamida, analisando as alterações locais, a segurança, a eficácia e as complicações deste método de tratamento. Casuística e métodos - Entre março de 1998 e maio de 2000, 58 pacientes com varizes de esôfago foram tratados com ligaduras com alças pré-atadas, confeccionadas com fio de poliamida (26 esquistossomóticos, 11 com cirrose alcoólica, 9 com hepatite C, 5 com hepatite B, 4 de causa não esclarecida, 2 com hepatite B e C e 1 com síndrome de Budd-Chiari; 42 pacientes eram do sexo masculino e 16 do sexo feminino, com média de idade de 47,67 $\pm 13,12$ anos). Confeccionou-se artesanalmente um canal acessório que acoplado à extremidade do endoscópio, permitia que alças pré-atadas feitas com fio de poliamida fossem conduzidas ao esôfago com auxílio de tubo metálico flexível para se proceder às ligaduras das varizes esofágicas. No total, 506 ligaduras foram realizadas, distribuídas por 223 sessões (média 2,26 $\pm 1,08$ ligaduras por sessão). As sessões foram realizadas com intervalos de 15 dias até a completa erradicação das varizes esofágicas. A ecoendoscopia foi utilizada como método complementar para avaliar a erradicação das varizes em 10 doentes. Resultados - O procedimento foi realizado com sucesso em todos os pacientes. A completa erradicação das varizes foi obtida em 47 (81,03\%) doentes. Em 37 (63,79\%) as ligaduras resultaram em pseudopólipos. Não foram identificados complicações sistêmicas ou óbitos. A ecoendoscopia mostrou trombose nos pseudopólipos formados. No período de seguimento que variou de 4 a 32 meses, observou-se recidiva das varizes em $9(15,51 \%)$ pacientes, tratados com êxito por nova ligadura com fio de poliamida em 5 pacientes e esclerose endoscópica em 4 . O custo médio de cada ligadura foi estimado em R \$3,60. Conclusões - O tratamento endoscópico, utilizando ligadura com fio de poliamida, revelou ser método seguro, eficaz, de simples realização e de baixo custo, no tratamento das varizes esofágicas. Esse método demonstrou também um novo aspecto que é a obliteração das varizes esofágicas, pelos pseudopólipos, sem necrose.

DESCRITORES - Varizes esofágicas e gástricas. Hemorragia gastrointestinal. Ligadura. Técnicas de sutura. Endoscopia do sistema digestório.

\section{INTRODUÇÃO}

A esclerose endoscópica e a ligadura com bandas elásticas tornaram-se métodos com bases técnicas bem estabelecidas no controle da hemorragia aguda e na prevenção da recidiva do sangramento de varizes esofágicas.

Estudos comparando a esclerose endoscópica com as ligaduras elásticas revelaram que o método de ligadura elástica é tão eficaz quanto o da esclerose endoscópica no controle do sangramento das varizes esofágicas, apresentando, contudo, as vantagens da erradicação mais rápida das varizes, em menor número de sessões e com menores índices de complicações ${ }^{(1,3,7,12)}$.

Os fenômenos isquêmicos relacionados ao uso das bandas elásticas, com necrose no local da ligadura e formação de úlceras, responsáveis eventuais por

Serviço de Endoscopia Geral, Hospital Sírio Libanês, São Paulo, SP.

Endereço para correspondência: Dr. Francisco Susumu C. Koyama - Av. 11 de junho, 730 - apt.43 - Liberdade - 04041-002 - São Paulo, SP. E-mail: drsk@ig.com.br 
quadro de sangramento maciço, principalmente em pacientes com a função hepática gravemente comprometida ${ }^{(11)}$, ainda permanecem como importante inconveniente desse método de tratamento, mesmo com os dispositivos mais modernos de múltiplas ligaduras. Esse fato, associado ao elevado custo desses dispositivos, fez com que em alguns centros, o tratamento de escolha continue sendo a esclerose.

A procura de uma técnica que possa reunir critérios de segurança, eficácia, facilidade de realização, baixo custo e complicações mínimas são desejados por todos os endoscopistas. Com este intuito, foi desenvolvido um método alternativo para o tratamento das varizes esofágicas, no qual o cordão varicoso é tratado, utilizando-se alça pré-atada com ligadura de fio de poliamida (LFP)

O objetivo do presente estudo foi descrever e avaliar o tratamento das varizes esofágicas, pelo método de ligadura com alça pré-atada, confeccionada com fio de poliamida, analisando-se as alterações nos locais das suas aplicações no esôfago e seus resultados em termos de segurança, eficácia e presença de complicações.

\section{CASUÍSTICA E MÉTODOS}

Entre março de 1998 e maio de 2000, 58 pacientes consecutivos foram tratados com ligaduras, utilizando-se alças pré-atadas confeccionadas com fio de poliamida. Quarenta e dois (72,41\%) pacientes eram do sexo masculino e $16(27,58 \%)$ do feminino, média de idade de 47,67 $\pm 13,12$ anos, mediana de 46 , variando de 16 a 74 anos. Vinte e seis pacientes apresentavam como causa da hipertensão portal e das varizes esofágicas a esquistossomose, 11 a cirrose alcoólica, 9 a hepatite $\mathrm{C}, 5$ a hepatite B, 2 as hepatites B e C, 1 a síndrome de Budd-Chiari e em 4 a causa não foi esclarecida.

Foram incluídos neste estudo todos os pacientes com hipertensão portal e varizes de esôfago com histórico de sangramento prévio ou que apresentavam varizes de grosso calibre com os chamados sinais de grande potencial de sangramento, ao exame endoscópico. Adotou-se como critério de exclusão os pacientes com mais de uma sessão de tratamento endoscópico das varizes esofágicas, independentemente do método utilizado.

Trinta e quatro $(58,62 \%)$ pacientes já haviam apresentado pelo menos um episódio de sangramento decorrente da ruptura das varizes esofágicas e $24(41,37 \%)$ foram tratados de modo profilático, por apresentarem varizes de grosso calibre com sinais de grande potencial de sangramento.

Representando diferentes situações de gravidade 33 (56,90\%) pacientes com cirrose foram classificados como Child A, 21 (36,20\%) como Child B e 4 (6,90\%) como Child C, de acordo com a classificação de Child-Turcotte, modificada por Pugh em 1973. Todos os 26 pacientes esquistossomóticos apresentavam função hepática preservada.

As varizes observadas durante o procedimento endoscópico foram classificadas de acordo com a Japanese Research Society for Portal Hypertension em função de sua cor, sinais de cor vermelha, forma e localização ${ }^{(5)}$. A classificação endoscópica das varizes dos 58 pacientes envolvidos neste estudo pode ser observada no Quadro 1.

\begin{tabular}{|lcc|}
\hline Classificação & $\mathbf{n}$ & $\%$ \\
\hline CbLiF1Rc ( - ) & 02 & 3,45 \\
CbLiF2Rc ( - ) & 11 & 18,96 \\
CwLiF1Rc ( + & 03 & 5,17 \\
CwLiF2Rc ( - ) & 08 & 13,80 \\
CwLiF2Rc ( + & 05 & 8,62 \\
CwLiF2Rc ( ++ ) & 04 & 6,90 \\
CwLiF2Rc ( +++ ) & 01 & 1,72 \\
CwLiF3Rc ( + ) & 03 & 5,27 \\
CwLiF3Rc ( ++ ) & 08 & 13,96 \\
CwLiF3Rc ( +++ ) & 13 & 22,41 \\
Total & 58 & 100 \\
\hline Fonte: Arquivo do Setor de Endoscopia Geral do Hospital Sírio-libanês
\end{tabular}

QUADRO 1 - Classificação endoscópica das varizes dos 58 pacientes estudados

As LFP foram realizadas em todos os pacientes pelo mesmo endoscopista, com ampla experiência em procedimentos endoscópicos diagnósticos e terapêuticos. Considerou-se como ressangramento, episódio de melena e/ou hematêmese durante o tratamento e, como complicação, as intercorrências no mesmo período.

O protocolo deste estudo foi aprovado pelo Comitê de Ética em Pesquisa do Hospital Sírio Libanês, São Paulo, SP. Os pacientes foram previamente cientificados do procedimento a que seriam submetidos e consentiram em atender ao protocolo exposto.

Os procedimentos foram realizados com videoendoscópios de visão frontal da marca Olympus, modelo GIF-130 e fibroendoscópios modelos XQ10 e XQ20 (Olympus Optical Co., Ltda., Miami, EUA).

Confeccionou-se artesanalmente um dispositivo de ligadura para fios (DLF) para ser acoplado à extremidade distal dos endoscópios de visão frontal, constituído de um adaptador para ligaduras elásticas seqüenciais (Wilson-Cook Medical, Winston-Salem, NC, EUA), que foi fixado a um tubo plástico com cerca de $60 \mathrm{~cm}$ de comprimento e diâmetro de $3 \mathrm{~mm}$ (Figura 1-A).

Um tubo metálico flexível, em forma de mola, foi usado para conduzir em sua extremidade uma alça pré-atada confeccionada com de fio de poliamida de $0,37 \mathrm{~mm}$ de espessura (Figura 1-B). Utilizou-se tesoura endoscópica de fechamento lateral da marca Olympus, modelo FS-3L1 e FS-1K(Olympus Optical Co., Ltda., Shijuku-Tokyo, Japão) para secção do fio.
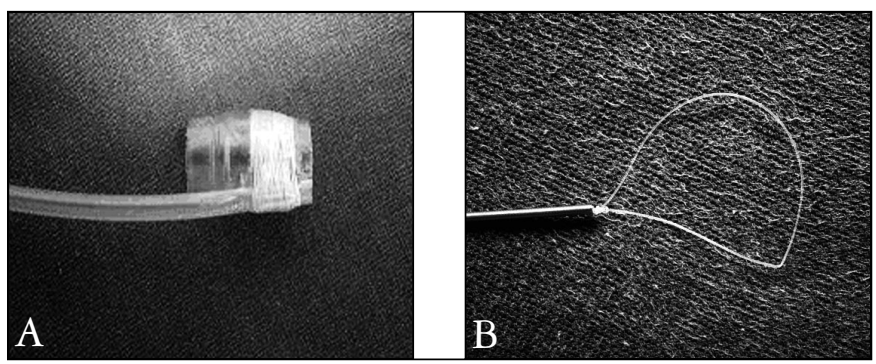

FIGURA 1 - A) DFL após sua montagem

B) Alça de poliamida na extremidade do tubo metálico 


\section{Técnica}

Após exame detalhado do esôfago, estômago e duodeno procedia-se à conexão do DLF à extremidade distal do endoscópico. Posicionava-se o endoscópio no segmento do órgão com varizes a serem tratadas. Exteriorizava-se, através dos DLF, a alça préatada com cerca de $3,0 \mathrm{~cm}$ de comprimento empurrado pelo tubo metálico flexível na luz esofágica. O fio de poliamida era, então, tracionado para ampliar o espaço no interior da alça e diminuir o seu tamanho, fazendo com que a mesma se adapte quase que de forma perfeita ao dispositivo de ligadura. Realizava-se, então, o contato frontal de $360^{\circ}$ entre o ponto de aplicação e a face distal do DLF, acionando-se o mecanismo de aspiração do endoscópio para que a variz e o tecido adjacente a mesma fossem aspirados para o interior do adaptador. A ligadura era aplicada pela tração manual vigorosa do fio de poliamida, pelo auxiliar por alguns segundos. Este procedimento fazia com que o nó fosse empurrado pelo tubo metálico até que ocorresse o estrangulamento do pseudopólipo formado. Ao término da ligadura, o fio de poliamida era cortado com tesoura endoscópica. Quando uma outra ligadura era necessária, renovava-se apenas a alça de poliamida, sem se retirar o aparelho do interior do esôfago. Esta segunda ligadura era sempre realizada em posição diferente da primeira.

Considerou-se sangramento conseqüente ao procedimento, episódios de hemorragia durante a realização das LFP ou sangramento no ponto de aplicação das mesmas, independentes do tempo decorrido após as ligaduras.

\section{Seguimento}

Os doentes foram acompanhados por 4 a 32 meses, com média de 18,75 $\pm 8,73$ meses. Exames endoscópicos de controle foram realizados em intervalos de 4 a 6 meses. As varizes foram consideradas erradicadas quando não eram mais observados os cordões varicosos. A recidiva das varizes foi tratada, quando possível, de modo similar ao tratamento inicial. Ao término do tratamento, 10 pacientes com pseudopólipos realizaram controle da erradicação pela ecoendoscopia com aparelho radial da marca Olympus, modelo GF-UM20 (Olympus Optical Co., Ltda., Tokyo, Japão) e transdutores de 7,5 e $12 \mathrm{MHz}$.

\section{RESULTADOS}

Foram realizadas 223 sessões de LFP em 58 pacientes, média de 3,84 $\pm 1,39$ sessões, variando de 2 a 7 sessões por paciente. $\mathrm{O}$ número total de ligaduras aplicadas foi de 506 , média de $2,26 \pm 1,08$ ligaduras, variando de 1 a 6 ligaduras por sessão.

No $15^{\circ}$ dia, o exame endoscópico revelou que 132 ligaduras com LFP, ainda permaneciam na base dos pseudopólipos. Houve redução do calibre das varizes esofágicas em todos os doentes. A completa erradicação das varizes foi obtida em 47 (81,03\%) pacientes. Pseudopólipos foram evidenciados em $37(63,79 \%)$ pacientes e na sua ausência, observou-se no ponto de ligadura a presença de pequena cicatriz branca. Em outros três $(5,17 \%)$ pacientes pequenas úlceras, rasas e ovaladas foram identificadas, cicatrizando completamente após 2 semanas sem o uso de qualquer medicação. A Figura 2 mostra os pseudopólipos.

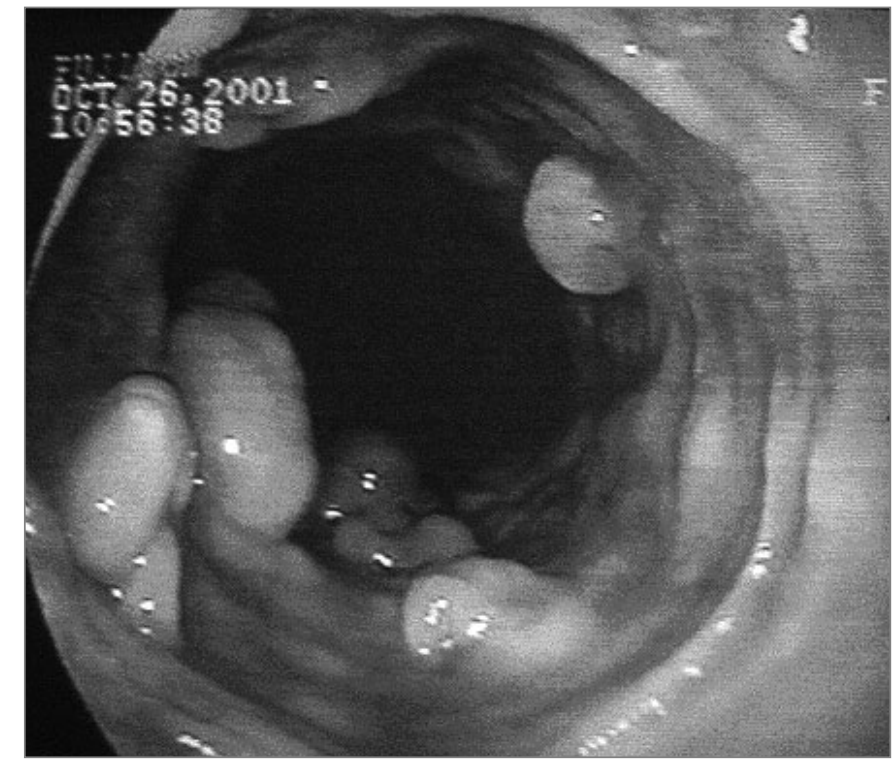

FIGURA 2 - Pseudopólipos formados após as ligaduras

Durante o período de seguimento, observou-se em todos os casos, diminuição gradativa do tamanho dos pseudopólipos, sem alcançar total desaparecimento. As ligaduras desapareceram em sua totalidade.

Ao término do tratamento pôde-se identificar o desaparecimento dos sinais de cor vermelha, o endurecimento do cordão varicoso à manipulação com instrumental e a interrupção das varizes próximas aos pseudopólipos. A ecoendoscopia radial foi realizada em 10 doentes, demonstrando formações hiperecóicas no interior dos pseudopólipos, sugerindo, juntamente com os achados mencionados acima, a trombose das varizes.

A recidiva das varizes foi observada em nove $(15,51 \%)$ doentes. Novo procedimento com LFP foi realizado em cinco $(8,62 \%)$ pacientes e em quatro $(6,89 \%)$ as varizes foram tratadas por esclerose, por serem de diminuto calibre. Em todos os doentes as varizes foram novamente erradicadas.

Sangramento maciço ocorreu em um $(1,72 \%)$ paciente, devido à tração súbita no tubo metálico, ocasionando avulsão do pseudopólipo e laceração da mucosa adjacente. A hemorragia foi controlada pela aplicação de ligaduras com fio de poliamida em pontos proximal e distal ao sangramento. Complicações de menor gravidade foram observadas e podem ser analisadas na Tabela 1. Não foram identificadas complicações sistêmicas ou óbitos.

TABELA 1 - Complicações associadas ao tratamento das varizes esofágicas com alça pré-atada confeccionada com fio de poliamida em 58 pacientes

\begin{tabular}{lc}
\hline Complicações & $\mathbf{n}(\%)$ \\
\hline Úlceras & $21(36,20 \%)$ \\
Disfagia & $08(13,80 \%)$ \\
Dor epigástrica & $05(8,62 \%)$ \\
Ressangramento & $03(5,17 \%)$ \\
Estenose & $01(1,72 \%)$ \\
\hline
\end{tabular}


Dor epigástrica e/ou retroesternal foi relatada em graus variados de intensidade, em cinco $(8,62 \%)$ pacientes. Em todos os casos, houve melhora sensível, após o uso de analgésicos (dipirona), e regressão completa no período máximo de 24 horas. A disfagia ocorreu em oito $(13,80 \%)$ doentes, na sua maioria de forma transitória, regredindo após o $3^{\circ}$ dia do tratamento. Estenose foi diagnosticada em um $(1,72 \%)$ paciente que apresentou disfagia persistente após o término do tratamento. O exame endoscópico de controle, realizado no $4^{\circ}$ mês, revelou a presença de estenose anular localizada no esôfago distal. O tratamento foi realizado em sessão única de dilatação, com sondas de Savary-Guilard, evoluindo o paciente sem queixas. Úlceras, decorrentes da queda do tecido ligado, ocorreram em $21(36,20 \%)$ pacientes, identificadas, na maioria das vezes, por cicatrizes ovaladas e brancas no local da ligadura. Apenas em três pacientes, as úlceras necessitaram de tempo superior a 2 semanas para sua cicatrização. Ressangramento acometeu três $(5,17 \%)$ doentes, todos de leve intensidade, identificados por episódios de melena e/ou hematêmese, sem repercussão hemodinâmica ou necessidade de transfusão sangüínea. A endoscopia realizada nas primeiras 12 horas após o início do quadro, revelou como causa do sangramento, em dois $(3,44 \%)$ pacientes, úlceras rasas no local da ligadura. Em um $(1,72 \%)$, o sangramento originavase de varizes remanescentes ainda não erradicadas. Desses pacientes, um apresentava critérios clínicos e laboratorial para ser classificado como Child C e dois como Child B.

Foram gastos cerca de $\mathrm{R} \$ 28,50$ para a produção das alças de poliamida e confeccionar o DLF e mais R $\$ 1.800,00$ para aquisição da tesoura endoscópica. Desta forma, o custo médio de cada ligadura realizada com alça pré-atada confeccionada com fio de poliamida foi de aproximadamente $\mathrm{R} \$ 3,60$.

\section{DISCUSSÃO}

A introdução por STIEGMANN et al. ${ }^{(15)}$, em 1986, das ligaduras com bandas elásticas, representou importante avanço no tratamento das varizes esofágicas, por ser método de simples realização, apresentar menores índices de complicações e necessitar menor número de sessões para sua erradicação quando comparadas à esclerose ${ }^{(2,7,18)}$.

Os dispositivos de ligaduras elásticas, entretanto, apresentaram alguns inconvenientes como a necessidade de múltiplas intubações tornando indispensável o uso do "overtube", a restrição do campo visual durante o procedimento e a ocorrência de fenômenos isquêmicos no local da ligadura, com necrose, queda do tecido ligado e formação de úlceras responsáveis, algumas vezes, por ressangramentos ${ }^{(6)}$, bem como o custo elevado do dispositivo.

O desenvolvimento das ligaduras com alças pré-atadas revelou eficácia semelhante às ligaduras elásticas no tratamento das varizes esofágicas, porém com melhora da visibilidade durante o procedimento e menores $\operatorname{custos}^{(3,14,16)}$.

Entretanto, permaneceram como metas a serem suplantadas por este método, os fenômenos isquêmicos locais seguidos de necrose, a possibilidade de remoção de sangue e secreções da luz esofágica durante o procedimento e o custo que, apesar de menor, ainda permanece elevado.
$\mathrm{Na}$ tentativa de se obter método endoscópico eficiente, seguro, prático, com baixos índices de complicações e que permitisse sua utilização em qualquer centro, sem restrição pelo seu custo, desenvolveu-se o tratamento das varizes esofágicas pela técnica LFP.

Este método reúne os critérios de segurança, eficácia, boa visibilidade durante o procedimento e a possibilidade de se realizar múltiplas ligaduras com uma única passagem do endoscópio, além de superar as dificuldades na remoção de sangue e secreções da luz esofágica por utilizar canal acessório para a realização das ligaduras.

O custo também foi amplamente reduzido, sendo 12 vezes menor que os dispositivos de ligadura simples produzidos na Alemanha (GFE, Garbsen, Voerde) e 30 a 56 vezes menor que os de múltiplas ligaduras confecccionados nos EUA (Wilson Cook Médical, Winston-Salem, Noth Carolina).

Fato que despertou grande interesse neste estudo foi a formação de pseudopólipos nos pontos de aplicação das LFP, primeiramente porque o propósito do tratamento foi alcançado, ou seja, obliteração das varizes esofágicas, fato confirmado pela ultra-sonografia endoscópica que demonstrou trombose das varizes nos pseudopólipos e, em segundo, pela ausência de necrose tecidual, conseqüente de formação de úlceras que, às vezes, são responsáveis por episódios de sangramento.

A formação dos pseudopólipos provavelmente estaria relacionada ao baixo grau do gradiente de pressão no momento em que a alça de poliamida se estabiliza, associado à capacidade do nó de Röehder deslizar, retrogradamente de forma discreta, determinando ligadura de baixa pressão. Nesse caso, a isquemia induzida pela LFP, não é suficiente para estabelecer a necrose que ocorre na quase totalidade dos casos de ligaduras com bandas elásticas e alças pré-atadas.

Em estudo ainda não publicado realizado no Peru, também foi possível reproduzir os pseudopólipos com a utilização de ligaduras elásticas confeccionadas artesanalmente a partir de sondas uretrais de demora (Foley $\mathrm{N}^{\circ} 16$ ). Nessas ligaduras, o orifício interno é maior do que nas bandas elásticas disponíveis no mercado, ratificando que, quando as ligaduras são realizadas com baixo gradiente de pressão, podem não acontecer fenômenos isquêmicos suficientes para que se instale a necrose tissular. As ligaduras usadas no estudo peruano ainda apresentam um inconveniente: a facilidade com que as bandas elásticas com maior diâmetro desprendem-se após as ligaduras. Nos pacientes tratados com as LFP, não foram observados casos em que as ligaduras tenham escapado precocemente.

Esses resultados levaram a considerar que no tratamento das varizes esofágicas a obliteração dos cordões varicosos poderia ser obtida por método de estrangulamento que determinasse apenas interrupção do fluxo sangüíneo sem necrose.

Este aspecto parece importante, pois SAKAI et al. ${ }^{(11)}$, em 1994, relataram ressangramento em $50 \%$ pacientes classificados como Child $\mathrm{C}$ no $7^{\circ}$ dia após terapêutica com ligaduras com bandas elásticas. O ponto de sangramento em todos os pacientes foi identificado como sendo uma úlcera sobre as varizes no local de ligadura. A provável justificativa deste fato se deve a grave disfunção hepática com alteração dos fatores de coagulação, além do retardo na cicatrização por deficiência da síntese protéica. 
$\mathrm{Na}$ presente casuística, quatro pacientes classificados como Child $\mathrm{C}$ foram tratados com ligaduras usando alças pré-atadas confeccionadas com fio de poliamida, sem causar hemorragia relacionada à queda de escara. Apesar de pequena esta casuística, os pacientes classificados como Child $\mathrm{C}$ não representam contraindicação para este método de tratamento.

As ligaduras com bandas elásticas são extremamente práticas na sua realização; em mãos habilitadas o tempo para a realização de seis ligaduras com bandas elásticas é estimado ser entre 6 a 9 minutos, com os dispositivos de ligadura simples, decrescendo para 75 segundos com os dispositivos de múltiplas ligaduras $^{(10)}$. O tempo de procedimento com as LFP não foi mensurado, mas é claramente superior aos valores descritos para as ligaduras com bandas elásticas, porém não excedendo a 3 ou 4 vezes o tempo necessário para a realização de uma ligadura com banda elástica quando se utiliza o dispositivo de ligadura simples. Este fato não parece ser de grande importância e que invalide esta técnica de tratamento para as varizes esofágicas, pois nem sempre é conveniente trocar o fator tempo por outros como segurança, eficácia, baixo índice de complicações e custo.

Outro método que objetiva a oclusão das varizes de esôfago por compressão local foi descrito por OHNUMA et al. ${ }^{(9)}$, em 1997. Esses autores utilizaram "clips" metálicos no tratamento de varizes esôfago-gástricas em crianças. À semelhança das LFP, os "clips" provocam oclusão das varizes sem que ocorra necrose e formação de úlceras.

Apesar do método descrito por OHNUMA et al. ${ }^{(9)}$ ter se revelado seguro e prático, apresentando mínimas complicações locais e ausência de complicações sistêmicas, é método de custo elevado, tornando quase que proibitivo o seu uso em muitas regiões. Esta parece ser outra grande vantagem das LFP uma vez que a maior parte do equipamento pode ser fabricada artesanalmente e as alças confeccionadas com fio de poliamida, que nada mais é que fio de pesca encontrado facilmente e de baixo custo, além do que, os DLF podem ser reaproveitados em sessões subseqüentes, por não serem descartáveis.

O método descrito por OHNUMA et al. ${ }^{(9)}$ apresenta ainda duas outras desvantagens: a primeira é a utilização do canal de trabalho do endoscópio para a realização das ligaduras, o que impossibilita a remoção do sangue e das secreções da luz esofágica durante o procedimento. A segunda é a taxa elevada de recidiva das varizes, atingindo índices de $67 \%$ no período de seguimento médio de 7,9 meses. Neste aspecto é muito superior à alcançada com o método de LFP que foi de $15,51 \%$, no período de seguimento médio de 18,75 meses. Deste modo, exige vigilância mais freqüente e maior número de sessões adicionais durante o seguimento, o que encarece ainda mais o procedimento.
Um método que levasse à trombose das varizes esofágicas sem necrose tecidual seria altamente desejável, já que ficaria afastada a possibilidade de sangramento por solução de continuidade da mucosa nos dias subseqüentes ao tratamento endoscópico. Isso parece ocorrer nos pacientes tratados pela LFP em que se obteve a formação dos pseudopólipos. Entretanto, essa vantagem ainda não é oferecida sistematicamente pelo método, pois falta orientação técnica que permita que todas as LFP resultem em pseudopólipo. O estabelecimento dessa orientação permite até considerações de grande interesse terapêutico, pois essa nova forma de tratamento poderia ser, talvez, aplicada aos pacientes com varizes esôfagogástricas que ainda não apresentaram sangramento.

A profilaxia ao primeiro sangramento por meio de procedimentos endoscópicos é tema controverso, não sendo aceita por todos os pesquisadores, pois algumas vezes os efeitos adversos são maiores do que os seus benefícios ${ }^{(4,8,17)}$.

As ligaduras com bandas elásticas, embora efetivas e com menores taxas de complicações do que a esclerose, estão indicadas nos pacientes que não toleram o tratamento medicamentoso com o uso dos betas bloqueadores e/ou nitratos, nos que apresentam contra-indicação ao seu uso ou não respondem a este método terapêutico ${ }^{(17)}$.

As LFP podem ser o estágio inicial de um método que também apresente valor na profilaxia ao primeiro sangramento, por ser efetivo, de fácil realização, baixo custo e principalmente, por determinar obliteração das varizes com mínima agressão local e ausência de complicações sistêmicas. Nestas condições, as contraindicações na sua aplicação seriam próprias do procedimento endoscópico em si.

A adesão e a tolerabilidade ao tratamento parecem ser melhores com as LFP do que com tratamento medicamentoso, além da resposta ser mais homogênea, ao contrário do tratamento farmacológico, para o qual apenas um terço dos pacientes responde favoravelmente ${ }^{(13)}$.

Entretanto, as LFP apresentam desvantagens em relação à terapia farmacológica, por agirem apenas localmente, não interferindo em outros órgãos e sistemas, como é o caso da gastropatia hipertensiva, além de necessitarem, à semelhança das ligaduras com bandas elásticas, de endoscopias de controle para a identificação e tratamento das varizes neoformadas.

A ligadura com alça pré-atada confeccionada com fio de poliamida é método ainda em desenvolvimento uma vez que não foi possível reproduzir de forma inexorável, pseudopólipos em todas as sessões. Mesmo nesta fase inicial do seu desenvolvimento revelou ser método útil no tratamento das varizes esofágicas, apresentando resultados que se sobrepõem aos das ligaduras com bandas elásticas.

Estudos randomizados confrontando a LFP com os atuais métodos de tratamento estão em andamento e brevemente poderão ser apreciados. 
Koyama FSC, Hashiba K, Bromberg SH, Cappelanes CA. Endoscopic treatment of esophageal varices, using pretied loop made with polyamide thread. Arq Gastroenterol. 2006;43(4):328-33.

ABSTRACT - Background - The elastic band ligation is the method of choice for treatment of esophageal varices. The action mechanism is a mechanical varices compression with thromboses. Based on this concept we developed a ligature method using pretied loop made with polyamide thread for the treatment of esophageal varices. Objective - The present study describes and evaluates the feasibility of the treatment of esophageal varices by the ligature method using pretied loop made with polyamide thread and analyzes the local changes of the ligations and the results, concerning safety, efficiency and complications of this procedure. Patients and methods - Between March, 1998 and May, 2000, 58 patients with esophageal varices were treated with pretied loop, made with polyamide thread (26 patients with schistosomiasis, 11 with alcoholic cirrhosis, 9 with hepatitis C, 5 with hepatitis B, 4 of unknown etiology, 2 with hepatitis B and C, and one with Budd-Chiari syndrome/ 42 men and 16 women/ average age of 47,67 $\pm 13,12$ years, range 16-74). A plastic tube was attached to the endoscope tip featured as an accessory working channel, allowing the pretied loop made with polyamide thread to be conducted to the esophagus facilitated by a flexible metallic tube, to perform the esophageal varices ligature. A total of 506 ligatures were done, distributed in 223 sections (average of 2,26 $\pm 1,08$ ligature, varying from 1 to 6 per section). The sessions were perform with the interval of 15 days, until the complete eradication of the esophageal varices. The ecoendoscopy was used as a complementary method to evaluate the varices eradication. Results - The esophageal varices were treated successfully in all patients. The complete eradication of varices was achieved in $47(81,03 \%)$ patients. In $37(63,79 \%)$ patients the ligatures resulted in pseudopolyps. It was not identified systemic complications or obits. The ecoendoscopy showed thrombosis in the pseudopolyps of 10 patients. The follow-up period was from 4 to 32 months and recurrence of the esophageal varices was observed in $9(15,51 \%)$ patients. The average cost of each ligature was estimated in US\$ 1,00 . Conclusion - The study indicates that endoscopic treatment using polyamide thread ligature is a safe, efficient, accessible and low cost method for treatment of esophageal varices treatment, demonstrating a new aspect, that is the obliteration of varices without necrosis, by formation of pseudopolyps.

HEADINGS - Esophageal and gastric varices. Gastrointestinal hemorrhage. Ligation. Suture techniques. Endoscopy, digestive system.

\section{REFERÊNCIAS BIBLIOGRÁFICAS}

1. Baroncini D, Milandri GL, Borioni D, Piemontese A, Cennamo V, Billi P, Dal Monte PP, D’Imperio ND. A prospective randomized trial of sclerotherapy versus ligation in the elective treatment of bleeding esophageal varices. Endoscopy. 1997;29:235-40.

2. Gimson AE, Ramage JK, Panos MZ, Hayllar K, Harrison PM, Williams R, Westaby D. Randomized trial of variceal banding ligation versus injection sclerotherapy bleeding oesophageal varices. Lancet. 1993;342:391-4

3. Hepworth CC, Burnham WR, Swain CP. Development and application of endoloops fo the treatment of bleeding esophageal varices. Gastrointest Endosc. 1999;50:677-84

4. Jalan R, Hayes PC. UK guidelines on the management of variceal haemorrhage in cirrhotic patients. Gut. 2000;46(Suppl 3-4):III1-III5.

5. Japanese Research Society for Portal Hypertension. The general rules for recording endoscopic findings on esophageal varices. Jp J Surg.1980;10:84-7.

6. Johnson PA, Campbell DR, Antonson CW, Weston AP, Shuler FN, Lozoff RD. Complication associated with endoscopic band ligation of esophageal varices. Gastrointest Endosc. 1993;39:181-5.

7. Laine L, El Newihi HM, Migikovsky B, Sloane R, Garcia F. Endoscopic ligation compared with sclerotherapy for the treatment of bleeding esophageal varices. Ann Intern Med. 1993;119:1-7.

8. Lebrec D. Primary prevention of variceal bleeding. What's new? Hepatology. 2001;33:1003-4

9. Ohnuma N, Takahashi H, Tanabe M, Yoshida H, Iwai J, Muramatsu T. Endoscopic variceal ligation using a clipping apparatus in children with portal hypertension. Endoscopy. 1997;29:86-90.
10. Saeed ZA. The Saeed six-shooter: a prospective study of a new endoscopic multiple rubber-band ligation for the treatment of varices. Endoscopy. 1996;28:556-64.

11. Sakai P, Maluf-Filho F, Melo JM, Ishioka S. Is endoscopic band ligation of esophageal varices contraindicated in Child-Pugh C patients? Endoscopy. 1994;26:511-2.

12. Sarin SK, Govil A, Jain AK, Guptan RC, Issar SK, Jain M, Murthy NS. Prospective randomized trial of endoscopic sclerotherapy versus variceal band ligation for esophageal varices: influence on gastropathy, gastric varices and variceal recurrence. Hepatology. 1997;26:826-32.

13. Seewald S, Seitz U, Yang M, Soehendra N. Variceal bleeding and portal hypertension: still a therapeutic challenge? Endoscopy 2001; 33: 126-39.

14. Shim CH, Cho JY, Park YJ, Kim YS, Kim YT,Hong SJ, Moon JH, Cho YD, Kim JO, Kim YS, Lee JS, Lee MS. Mini-detachable snare ligation for the treatment of esophageal varices. Gastrointest Endosc. 1999;50:673-7.

15. Stiegmann GV, Cambre T, Sun JH. A new endoscopic elastic band ligating device. Gastrointest Endosc. 1986;32;230-2.

16. Sung JJY, Chung SCS. The use of detachable mini-loop for the treatment of esophageal varices. Gastrointest Endosc. 1998:47:178-81.

17. Vlachogiannakos J, Goulis J, Patch D, Burroughs AK. Primary prophylaxis for portal hypertensive bleeding in cirrhosis. Aliment Pharmacol Ther. 2000;14:851-60.

18. Young MF, Sanowski RA, Rasche R. Comparison and characterization of esophageal varices versus endoscopic sclerotherapy. Gastrointest Endosc. 1993;39:119-22.

Recebido em 14/2/2005. Reapresentado em 19/4/2006. Aprovado em 12/5/2006. 